(95.2\%) underwent respiratory polygraphy (377 inpatient, 99 at home) and 24 (4.8\%) overnight oximetry. Initial mean ESS ( \pm SD) was $13.3 \pm 6.0$ and was positive $(=10)$ in 354 patients $(70.8 \%)$. SDB was demonstrated in 309 patients (61.8\%) and periodic limb movements in 8. Positivity rates varied with referral source (GP-64.8\%, diabetes-55.2\%, respiratory-67.6\%, ENT - 55.2\%) and sleep study type (inpatient polygraphy- $60.2 \%$, home polygraphy-76.8\%, oximetry-58.3\%). Diagnosis was obstructive sleep apnoea (OSA) ( $n=246,15$ with coexistent hypoventilation), upper airways resistance $(n=56)$, obesity hypoventilation $(n=4)$, central sleep apnoea $(n=2)$ and COPD-related nocturnal hypoxia $(n=1)$. Median ODI for all studies was 9 (range 0-150). OSA was mild in 72 patients (29.3\%), moderate in $65(26.4 \%)$ and severe in 106 (43.1\%). ODI was $<5$ in 3 patients $(1.2 \%)$ but the study deemed positive. Following clinical assessment, 269 patients (53.8\%) commenced CPAP, of whom $36(13.4 \%)$ had an initial ESS=9. In these patients, severity of SDB did not relate to baseline ESS (mean ODI $32.0(\mathrm{ESS}=9)$ vs $30.5 \mathrm{ESS}=10)$ ). Of the $106 / 500$ patients with $\mathrm{ESS}=9,54.3 \%$ had a positive study and $33.3 \%$ were commenced on CPAP, as compared to $65.5 \%$ and $58.8 \%$ respectively of patients with $\mathrm{ESS}=10$.

Conclusion We identified a reasonable percentage of patients referred with suspected SDB. A significant number of such patients had a normal ESS, which may underestimate symptoms warranting CPAP. A negative ESS should not preclude sleep referral and should be used with caution when designing referral criteria.

\section{P24 PREVALENCE OF OBSTRUCTIVE SLEEP APNOEA IN PATIENTS SCHEDULED FOR BARIATRIC SURGERY AND VALIDATION OF THE STOP-BANG QUESTIONNAIRE AS A SCREENING TOOL}

doi:10.1136/thoraxjnl-2011-201054c.24

R Kaiser, C Hammond, L Restrick, H Branley, S Lock, P Sufi, D Heath. Whittington Hospital, London, UK

Background Obstructive Sleep Apnoea (OSA) is common in morbidly obese patients scheduled for bariatric surgery, and many sleep centres perform routine preoperative sleep studies for all. However a significant proportion will not have significant OSA. Epworth Sleepiness Score (ESS) is unreliable in predicting the risk of OSA. A practical screening tool is ideally required. We aimed to define the prevalence of OSA in our bariatric patient population and validate the STOP-BANG questionnaire as a screening tool.

Methods Retrospective review of bariatric patients who had sleep studies over a 3-month period from January to March 2011. Clinical data collected from medical notes and sleep study results. STOPBANG scores derived retrospectively from clinical data. Questionnaire included 8 yes/no questions, scored 1 for every yes answer (Abstract P24 table 1). A score of 4 or more was considered as high risk for having OSA. STOP-BANG scores were then correlated with

Abstract P24 Table 1 Stop-bang questionnaire (Score 1 for every Yes answer)

\begin{tabular}{lc}
\hline Do you snore loudly to be heard behind closed doors? & Yes/No \\
Do you feel tired, fatigued, or sleepy during daytime? & Yes/No \\
Has anyone observed you stop breathing during sleep? & Yes/No \\
Do you have or are you being treated for high blood pressure? & Yes/No \\
BMl $>35 \mathrm{~kg} / \mathrm{m}^{2} ?$ & Yes \\
Neck circumference $>40 \mathrm{~cm}$ & Yes/No \\
Gender Male? & Yes/No
\end{tabular}

If Score 4 or more $=$ high risk of OSA

If Score 3 or less $=$ low risk of OSA sleep study results. Significant OSA (which may require treatment with preoperative CPAP) was defined as a Apnoea-Hypopnoea Index (AHI) of at least $>15$.

Results Patient characteristics ( $n=61)$ : mean age 45 (24-69), 87.3\% female, mean BMI 46.2 (35-67), mean ESS 6.5 (0-20). Sleep study results $-18 \%$ had AHI $15-30,13.1 \%$ had AHI $>30.55 .7 \%$ had STOP-BANG score of $=4,44.3 \%$ had score of $=3$. Of patients with significant OSA (AHI>15): mean BMI 47.2, mean neck circumference 41.4 (SD 2.5), Mean ESS 8.5 (SD 4.84), 66.6\% had ESS $<11$, $89.4 \%$ were loud snorers. Using STOP-BANG score of $=4$ to screen for OSA with AHI $>15$-Sensitivity $94.7 \%$, Specificity $61 \%$, positive predictive value $52.9 \%$, negative predictive value $96.2 \%$.

Conclusions $31 \%$ of patient population studied had at least moderate OSA. ESS poorly predictive of risk of OSA. Using a high risk STOP-BANG score of 4 had a high sensitivity but poor specificity. However, a low risk score of $<4$ had a high negative predictive value of $96.2 \%$ for AHI $>15$. Therefore STOP-BANG questionnaire using a cut-off risk score of 4 can be used as a screening tool to rule out significant OSA and thus avoiding sleep studies in a significant proportion of low risk patients.

\section{P25 A NOVEL COST-SAVING APPROACH TO THE SLEEP CLINIC NON-ATTENDERS WITH CPAP MACHINES}

doi:10.1136/thoraxjnl-2011-201054c.25

A H Shah, P Close, J Oliver, S D West. Regional Sleep Service, Department of Respiratory Medicine, Freeman Road Hospital, Newcastle-upon-Tyne, UK

Introduction Increasing numbers of patients are diagnosed with Obstructive Sleep Apnoea Syndrome (OSAS) and require Continual Positive Airway Pressure (CPAP) treatment. There is little work following up patients who have received CPAP machines and then default from outpatient review. Contacting these patients may allow their care to be optimised and reclaiming unused machines may enable cost-savings in terms of reuse. Our objective was to establish if Sleep Clinic non-attenders were still using their CPAP machines, and whether a potential cost-saving was achievable from reclamation of CPAP machines.

Methods A search was performed on the Sleep Service CPAP database for patients with OSAS who had defaulted from follow-up for at least 3 years. Administration staff performed phone-based interviews based on a simple proforma, which established the status of patients' CPAP use-active, usage with problems, or no longer using. Sleep Service physiologists contacted the patients having problems with CPAP, to troubleshoot and arrange appointments for review and machine servicing. Arrangements were made for unused CPAP machines to be returned. A cost analysis was based on cost of a CPAP unit and the overtime cost of the administration staff involved in contacting patients.

Results We identified 196 patients who had CPAP machines and had defaulted from follow-up for 3 years or more. Of these, 138 (70\%) patients stated they wished to continue CPAP treatment and required out-patient review. There were 58 (30\%) patients no longer using CPAP and wanted to discontinue; they were asked to return their CPAP machine or be invoiced. Machines in good condition could be re-used for other patients. Based on unit cost, this could represent savings of up to $£ 10400$. Administration overtime staff costs for this project were $£ 386$ ( $44 \mathrm{~h}$ work) and therefore the overall potential cost-saving was $£ 10014$.

Conclusions An active search and contact of non-attenders to Sleep Clinic prescribed CPAP has identified a significant proportion no longer using their CPAP machines. The cost of this search was relatively low and thus cost-savings could be achieved in terms of reclaiming and reusing machines. This may represent an important cost-saving exercise as Sleep services continue to expand. 